PROCEEDINGS OF THE

AMERICAN MATHEMATICAL SOCIETY

Volume 139, Number 9, September 2011, Pages 3279-3290

S 0002-9939(2011)10768-8

Article electronically published on February 9, 2011

\title{
TIME-FREQUENCY CONCENTRATION OF GENERATING SYSTEMS
}

\author{
PHILIPPE JAMING AND ALEXANDER M. POWELL
}

(Communicated by Michael T. Lacey)

\begin{abstract}
Uncertainty principles for generating systems $\left\{e_{n}\right\}_{n=1}^{\infty} \subset L^{2}(\mathbb{R})$ are proven and quantify the interplay between $\ell^{r}(\mathbb{N})$ coefficient stability properties and time-frequency localization with respect to $|t|^{p}$ power weight dispersions. As a sample result, it is proven that if the unit-norm system $\left\{e_{n}\right\}_{n=1}^{\infty}$ is a Schauder basis or frame for $L^{2}(\mathbb{R})$, then the two dispersion sequences $\Delta\left(e_{n}\right)$, $\Delta\left(\widehat{e_{n}}\right)$ and the one mean sequence $\mu\left(e_{n}\right)$ cannot all be bounded. On the other hand, it is constructively proven that there exists a unit-norm exact system $\left\{f_{n}\right\}_{n=1}^{\infty}$ in $L^{2}(\mathbb{R})$ for which all four of the sequences $\Delta\left(f_{n}\right), \Delta\left(\widehat{f_{n}}\right), \mu\left(f_{n}\right)$, $\mu\left(\widehat{f_{n}}\right)$ are bounded.
\end{abstract}

\section{INTRODUCTION}

The uncertainty principle in harmonic analysis states that a function $f$ and its Fourier transform $\widehat{f}(\xi)=\int f(t) e^{-2 \pi i t \xi} \mathrm{d} t$ cannot be simultaneously too well localized. Heisenberg's uncertainty principle offers a classical interpretation of this in terms of means and dispersions. Heisenberg's inequality states that for every $f \in L^{2}(\mathbb{R})$ with unit-norm $\|f\|_{2}=1$,

$$
\Delta(f) \Delta(\widehat{f}) \geq \frac{1}{4 \pi} .
$$

For unit-norm $f \in L^{2}(\mathbb{R})$, the mean $\mu(\cdot)$ is defined by $\mu(f)=\int t|f(t)|^{2} \mathrm{~d} t$ and the dispersion $\Delta(\cdot)$ is defined by $\Delta(f)=\left(\int|t-\mu(f)|^{2}|f(t)|^{2} \mathrm{~d} t\right)^{1 / 2}$. It is common to say that $f$ is mostly concentrated in the time-frequency plane in a Heisenberg box centered at $(\mu(f), \mu(\widehat{f}))$ with side lengths determined by $\Delta(f)$ and $\Delta(\widehat{f})$. Heisenberg's inequality states that this box has area at least $1 /(4 \pi)$. For a survey on uncertainty principles, see [10].

Heisenberg's uncertainty principle applies to individual functions $f \in L^{2}(\mathbb{R})$. However, there are also versions of the uncertainty principle that constrain the

Received by the editors February 22, 2010 and, in revised form, August 23, 2010.

2010 Mathematics Subject Classification. Primary 42A38, 42A65.

Key words and phrases. Compactness, exact system, frame, Schauder basis, time-frequency concentration, uncertainty principle.

The second author was supported in part by NSF Grant DMS-0811086. Portions of this work were completed during visits to the Université d'Orléans (Orléans, France), the Academia Sinica Institute of Mathematics (Taipei, Taiwan), and the City University of Hong Kong (Hong Kong, China). The second author is grateful to these institutions for their hospitality and support. The first and second authors were both partially supported by the ANR project AHPI Analyse Harmonique et Problèmes Inverses.

(c)2011 American Mathematical Society Reverts to public domain 28 years from publication 
collective time-frequency localization properties of systems of functions $\left\{e_{n}\right\}_{n=1}^{\infty} \subset$ $L^{2}(\mathbb{R})$ such as orthonormal bases. For example, the Balian-Low uncertainty principle (see [2]) states that if the Gabor system

$$
\mathcal{G}(f, 1,1)=\left\{f_{m, n}\right\}_{m, n \in \mathbb{Z}}=\left\{e^{2 \pi i m t} f(t-n)\right\}_{m, n \in \mathbb{Z}}
$$

is an orthonormal basis for $L^{2}(\mathbb{R})$, then the strong uncertainty constraint $\Delta(f) \Delta(\widehat{f})$ $=\infty$ must hold. The Balian-Low theorem depends crucially on the rigid structure of Gabor systems.

This article addresses uncertainty principles for general generating systems $\left\{e_{n}\right\}_{n=1}^{\infty}$ for $L^{2}(\mathbb{R})$. Our main results are motivated by a question of H.S. Shapiro, 222, that asks to characterize the sequences $\Delta\left(e_{n}\right), \Delta\left(\widehat{e_{n}}\right), \mu\left(e_{n}\right), \mu\left(\widehat{e_{n}}\right)$ that arise for orthonormal bases $\left\{e_{n}\right\}_{n=1}^{\infty}$ of $L^{2}(\mathbb{R})$. The following uncertainty principle for this setting was proven in [19]:

$$
\begin{aligned}
& \text { If }\left\{e_{n}\right\}_{n=1}^{\infty} \text { is an orthonormal basis for } L^{2}(\mathbb{R}) \text {, then the three } \\
& \text { sequences } \mu\left(e_{n}\right), \Delta\left(e_{n}\right), \Delta\left(\widehat{e_{n}}\right) \text { cannot all be bounded. }
\end{aligned}
$$

Further results of this type may be found in [14, 16. A main goal of this article is to pursue the investigation of this phenomenon for more general generating systems in $L^{2}(\mathbb{R})$ (such as Schauder bases, frames, or exact systems) and for generalized dispersions involving a $|t|^{p}$ power weight (instead of the standard $|t|^{2}$ weight).

Overview and main results. The paper is organized as follows. Section 2 gives some background on generating systems and generalized power weight dispersions. Section 3 discusses the role of compactness in time-frequency concentration results and contains useful lemmas.

Our first main result, Theorem 4.2, is proven in Section 4. Roughly stated, Theorem 4.2 shows that if a unit-norm system $\left\{e_{n}\right\}_{n=1}^{\infty} \subset L^{2}(\mathbb{R})$ satisfies

$$
\forall f \in L^{2}(\mathbb{R}), \quad A\left(\sum_{n=1}^{\infty}\left|\left\langle f, e_{n}\right\rangle\right|^{s}\right)^{1 / s} \leq\|f\|_{2} \leq B\left(\sum_{n=1}^{\infty}\left|\left\langle f, e_{n}\right\rangle\right|^{r}\right)^{1 / r},
$$

then for $q r>2$ (with $p, q>1$ and $1<r \leq 2 \leq s<\infty$ ) the generalized means and dispersions $\mu_{p}\left(e_{n}\right), \Delta_{p}\left(e_{n}\right), \Delta_{q}\left(\widehat{e_{n}}\right)$ cannot all be bounded. This improves the dispersion requirements in [19] and extends beyond orthonormal bases. For example, if $\left\{e_{n}\right\}_{n=1}^{\infty}$ is a Schauder basis or frame for $L^{2}(\mathbb{R})$, then the standard means and dispersions $\mu\left(e_{n}\right), \Delta\left(e_{n}\right), \Delta\left(\widehat{e_{n}}\right)$ cannot all be bounded.

Our second main result, Theorem [5.2, appears in Section 5 and shows that the phenomenon in Theorem 4.2 is not true for exact systems. In particular, Theorem 5.2 constructively proves that for any $p, q>1$ there exists a unit-norm exact system $\left\{e_{n}\right\}_{n=1}^{\infty}$ for $L^{2}(\mathbb{R})$ such that $\Delta_{p}\left(e_{n}\right), \mu_{p}\left(e_{n}\right), \Delta_{q}\left(\widehat{e_{n}}\right)$ and $\mu_{q}\left(\widehat{e_{n}}\right)$ are all bounded sequences.

\section{BACKGROUND}

2.1. Generating systems. Throughout this section let $H$ be a separable Hilbert space with norm $\|\cdot\|$. The collection $\left\{e_{n}\right\}_{n=1}^{\infty} \subset H$ is a frame for $H$ if there exist constants $0<A, B<\infty$ such that

$$
\forall f \in H, \quad A\|f\|^{2} \leq \sum_{n=1}^{\infty}\left|\left\langle f, e_{n}\right\rangle\right|^{2} \leq B\|f\|^{2} .
$$


A standard result in frame theory states that there exists an associated dual frame $\left\{\widetilde{e}_{n}\right\}_{n=1}^{\infty} \subset H$ such that the following expansions hold with unconditional convergence in $H$ :

$$
\forall f \in H, \quad f=\sum_{n=1}^{\infty}\left\langle f, e_{n}\right\rangle \widetilde{e}_{n}=\sum_{n=1}^{\infty}\left\langle f, \widetilde{e}_{n}\right\rangle e_{n} .
$$

In general, frames need neither be orthogonal nor provide unique representations. For example, a union of $k$ orthonormal bases for $H$ is a frame for $H$. For further background on frame theory see 4,6 .

The system $\left\{e_{n}\right\}_{n=1}^{\infty} \subset H$ is minimal if for every $N \in \mathbb{N}, e_{N} \notin \overline{\operatorname{span}}\left\{e_{n}: n \neq N\right\}$. If $\left\{e_{n}\right\}_{n=1}^{\infty}$ is a minimal system and is a frame for $H$, then we say that $\left\{e_{n}\right\}_{n=1}^{\infty}$ is a Riesz basis for $H$. If $\left\{e_{n}\right\}_{n=1}^{\infty}$ is minimal and is complete in $H$, then we say that $\left\{e_{n}\right\}_{n=1}^{\infty}$ is an exact system in $H$.

The system $\left\{e_{n}\right\}_{n=1}^{\infty} \subset H$ is a Schauder basis for $H$ if for every $f \in H$ there exists a unique sequence $\left\{c_{n}\right\}_{n=1}^{\infty} \subset \mathbb{C}$ such that $\lim _{N \rightarrow \infty}\left\|f-\sum_{n=1}^{N} c_{n} e_{n}\right\|=0$. Moreover, there exists an associated dual basis $\left\{\widetilde{e}_{n}\right\}_{n=1}^{\infty} \subset H$ so that the coefficients $c_{n}$ are given by $c_{n}=\left\langle f, \widetilde{e}_{n}\right\rangle$.

An important result of Gurariı and Gurariı̌, see [13, 21], states that if $\left\{e_{n}\right\}_{n=1}^{\infty}$ with $\left\|e_{n}\right\|=1$ is a Schauder basis for $H$, then there exist $1<r \leq 2 \leq s<\infty$ and constants $0<A, B<\infty$ such that

$$
\forall f \in H, \quad A\left(\sum_{n=1}^{\infty}\left|\left\langle f, e_{n}\right\rangle\right|^{s}\right)^{1 / s} \leq\|f\| \leq B\left(\sum_{n=1}^{\infty}\left|\left\langle f, e_{n}\right\rangle\right|^{r}\right)^{1 / r} .
$$

The original version of Gurariǐ and Gurariı̌'s result, 13, 21, is stated differently than (2.3) using coefficients $\left\langle f, \widetilde{e}_{n}\right\rangle$ with respect to the dual basis $\left\{\widetilde{e}_{n}\right\}_{n=1}^{\infty}$ associated to $\left\{e_{n}\right\}_{n=1}^{\infty}$. However, this is equivalent to (2.3) since the dual basis is itself a Schauder basis and since $\left\|e_{n}\right\|=1$ implies the existence of $C \geq 1$ such that $1 \leq$ $\left\|\widetilde{e}_{n}\right\| \leq C$ for all $n \in \mathbb{N}$; e.g., see [24].

We shall primarily be interested in the cases where $H=L^{2}(\mathbb{R})$ or where $H=$ $\overline{\operatorname{span}}\left\{e_{n}\right\}_{n=1}^{\infty}$ is the closed linear span of a collection $\left\{e_{n}\right\}_{n=1}^{\infty} \subset L^{2}(\mathbb{R})$. Since we shall investigate the applicability of (1.1) to systems such as frames, Schauder bases, and exact systems, it is useful to note the following inclusions among different types of generating systems in $L^{2}(\mathbb{R})$ :

\{orthonormal bases $\} \subsetneq\{$ Riesz bases $\} \subsetneq\{$ Schauder bases $\} \subsetneq\{$ exact systems $\}$,

$\{$ Riesz bases $\} \subsetneq\{$ frames $\} ; \quad$ frames $\} \nsubseteq\{$ Schauder bases $\} ;$ \{Schauder bases $\} \nsubseteq$ frames $\}$.

The condition (2.3) will serve as a key feature in our analysis. In particular, (2.3) holds for all Schauder bases, frames, Riesz bases and orthonormal bases, but does not in general hold for exact systems. See [24, 5, 17] for additional information on generating systems.

2.2. Generalized means and dispersions. The standard dispersion defined by

$$
\Delta(h)=\left(\int|t-\mu(h)|^{2}|h(t)|^{2} \mathrm{~d} t\right)^{1 / 2} \quad \text { and } \quad \mu(h)=\int t|h(t)|^{2} \mathrm{~d} t
$$

implicitly makes use of the $|t|^{2}$ power weight. We shall consider a generalized class of dispersions and associated means defined in terms of the $|t|^{p}$ power weight with $p>1$. 
Fix $p>1$ and $h \in L^{2}(\mathbb{R})$ with $\|h\|_{2}=1$ and define the function $\varphi(a)=\int \mid t-$ $\left.a\right|^{p}|h(t)|^{2} \mathrm{~d} t$. One easily checks that if $\varphi(a)<\infty$ for some $a \in \mathbb{R}$, then $\varphi: \mathbb{R} \rightarrow \mathbb{R}$ is continuous (in particular $\varphi(a)$ is finite for all $a \in \mathbb{R}$ ) and is strictly convex. For this, recall that for any fixed $t \in \mathbb{R}, a \mapsto|t-a|^{p}$ is a strictly convex function.

Assume that $\|h\|_{2}=1$ and $\int|t|^{p}|h(t)|^{2} \mathrm{~d} t<\infty$ so that $\varphi(a)<\infty$ for all $a \in \mathbb{R}$. It is straightforward to verify that $\lim _{|a| \rightarrow \infty} \varphi(a)=\infty$. Since $\varphi$ is continuous it follows that $\varphi$ attains a global minimum. Moreover, since $\varphi$ is strictly convex the global minimum is unique. We may thus define

$$
\Delta_{p}(h)=\min _{a \in \mathbb{R}}\left(\int|t-a|^{p}|h(t)|^{2} \mathrm{~d} t\right)^{1 / 2}
$$

and

$$
\mu_{p}(h)=\arg \min _{a \in \mathbb{R}}\left(\int|t-a|^{p}|h(t)|^{2} \mathrm{~d} t\right)^{1 / 2} .
$$

Note that $\mu_{p}(h)$ is uniquely defined. We refer to $\Delta_{p}(h)$ as the $p$-dispersion of $h$ and $\mu_{p}(h)$ as the $p$-mean of $h$. When $p=2$ it is straightforward to verify that the closed form expressions $\mu_{2}(f)=\mu(f)$ and $\Delta_{2}(f)=\Delta(f)$ from (2.4) hold.

If $p \leq 1$, then strict convexity no longer holds and the $p$-mean need not be uniquely defined. For example, if $h=2^{-1 / 2}\left(\chi_{[-2,-1]}+\chi_{[1,2]}\right)$, then every $a \in[-1,1]$ is a minimizer for $\varphi(a)=\int|t-a||h(t)|^{2} \mathrm{~d} t$. We will therefore restrict our attention to $p>1$.

We now state some useful results on $p$-means and $p$-dispersions.

Lemma 2.1. Let $p>1$. Suppose that $h \in L^{2}(\mathbb{R})$ satisfies $\|h\|_{2}=1$ and that $\Delta_{p}(h)$ is finite (and hence $\mu_{p}(h)$ is also finite). If $h_{s}$ is defined by $h_{s}(t)=h(t-s)$, then $\Delta_{p}\left(h_{s}\right)=\Delta_{p}(h)$ and $\mu_{p}\left(h_{s}\right)=\mu_{p}(h)+s$. Moreover, if $h$ is even or odd, then $\mu_{p}(h)=0$.

Proof. It is clear that $\Delta_{p}\left(h_{s}\right)=\Delta_{p}(h)$ and $\mu_{p}\left(h_{s}\right)=\mu_{p}(h)+s$. Next assume that $h$ is either even or odd, so that $|h|^{2}$ is even. The change of variable $t \mapsto-t$ gives

$$
\int\left|t-\mu_{p}(h)\right|^{p}|h(t)|^{2} \mathrm{~d} t=\int\left|t+\mu_{p}(h)\right|^{p}|h(t)|^{2} \mathrm{~d} t .
$$

Since $\mu_{p}(h)$ is the unique minimizer of $\varphi(a)=\int|t-a|^{p}|h(t)|^{2} \mathrm{~d} t$ we have $\mu_{p}(h)=$ $-\mu_{p}(h)$. Thus, $\mu_{p}(h)=0$.

In general, convergence in $L^{2}(\mathbb{R})$ does not imply convergence of $p$-dispersions. For example, if we define $\psi_{s}=(1-s)^{1 / 2} \chi_{[-1 / 2,1 / 2]}+s^{1 / 2} \chi_{\left[s^{-4}, 1+s^{-4}\right]}$ and $\psi=$ $\chi_{[-1 / 2,1 / 2]}$, then $\lim _{s \rightarrow 0} \psi_{s}=\psi$ holds in $L^{2}(\mathbb{R})$, but $\lim _{s \rightarrow 0} \mu_{2}\left(\psi_{s}\right)=\infty \neq 0=$ $\mu_{2}(\psi)$ and $\lim _{s \rightarrow 0} \Delta_{2}\left(\psi_{s}\right)=\infty \neq \Delta_{2}(\psi)$. However, the following holds.

Proposition 2.2. Let $p>1$. Suppose that $f, g \in L^{2}(\mathbb{R})$ satisfy $\|f\|_{2}=\|g\|_{2}=1$ and that $\Delta_{p}(f)$ and $\Delta_{p}(g)$ are finite. For each $0<\alpha<1$ define $h_{\alpha}=\frac{f+\alpha g}{\|f+\alpha g\|_{2}}$. Then

$$
\lim _{\alpha \rightarrow 0} \Delta_{p}\left(h_{\alpha}\right)=\Delta_{p}(f) \quad \text { and } \quad \lim _{\alpha \rightarrow 0} \mu_{p}\left(h_{\alpha}\right)=\mu_{p}(f) .
$$


Proof. The proof is divided into four steps:

Step I. We first show that

$$
\limsup _{\alpha \rightarrow 0} \Delta_{p}^{2}\left(h_{\alpha}\right) \leq \Delta_{p}^{2}(f)
$$

Since $\Delta_{p}(f), \Delta_{p}(g)$ are finite it follows that $\left|t-\mu_{p}(f)\right|^{p}|f(t)|^{2}$ and $\left|t-\mu_{p}(f)\right|^{p}|g(t)|^{2}$ are integrable. Since $h_{\alpha} \rightarrow f$ almost everywhere and $\left|h_{\alpha}\right|^{2} \leq 2 \frac{|f|^{2}+|g|^{2}}{(1-\alpha)^{2}}$, the dominated convergence theorem implies that

$$
\Delta_{p}^{2}\left(h_{\alpha}\right) \leq \int\left|t-\mu_{p}(f)\right|^{p}\left|h_{\alpha}(t)\right|^{2} \mathrm{~d} t \rightarrow \int\left|t-\mu_{p}(f)\right|^{p}|f(t)|^{2} \mathrm{~d} t=\Delta_{p}^{2}(f) .
$$

The assertion (2.5) follows.

Step II. We next show that if $\alpha$ is sufficiently small, then

$$
\left|\mu_{p}\left(h_{\alpha}\right)-\mu_{p}(f)\right| \leq 2\left[4 \Delta_{p}^{2}(f)\right]^{1 / p}
$$

To begin, by (2.5), take $\alpha$ sufficiently small so that $\Delta_{p}^{2}\left(h_{\alpha}\right) \leq 2 \Delta_{p}^{2}(f)$. Let $\eta=$ $\left[4 \Delta_{p}^{2}(f)\right]^{1 / p}$. Then

$$
\int_{\left|t-\mu_{p}(f)\right|>\eta}|f(t)|^{2} \mathrm{~d} t \leq \eta^{-p} \int_{\left|t-\mu_{p}(f)\right|>\eta}\left|t-\mu_{p}(f)\right|^{p}|f(t)|^{2} \mathrm{~d} t \leq \frac{\Delta_{p}^{2}(f)}{\eta^{p}}=\frac{1}{4}
$$

Similarly,

$$
\frac{1}{2} \leq 1-\frac{\Delta_{p}^{2}\left(h_{\alpha}\right)}{\eta^{p}} \leq \int\left|h_{\alpha}(t)\right|^{2} \mathrm{~d} t-\int_{\left|t-\mu_{p}\left(h_{\alpha}\right)\right|>\eta}\left|h_{\alpha}(t)\right|^{2} \mathrm{~d} t=\int_{\left|t-\mu_{p}\left(h_{\alpha}\right)\right| \leq \eta}\left|h_{\alpha}(t)\right|^{2} \mathrm{~d} t .
$$

Also note that

$$
\int_{\left|t-\mu_{p}\left(h_{\alpha}\right)\right| \leq \eta}\left|h_{\alpha}(t)\right|^{2} \mathrm{~d} t \leq(1-\alpha)^{-2}\left(\int_{\left|t-\mu_{p}\left(h_{\alpha}\right)\right| \leq \eta}|f(t)|^{2} \mathrm{~d} t+\alpha^{2}+2 \alpha\right) .
$$

Now, towards a contradiction, suppose that $\left|\mu_{p}\left(h_{\alpha}\right)-\mu_{p}(f)\right|>2 \eta$ holds. This would imply that

$$
\int_{\left|t-\mu_{p}\left(h_{\alpha}\right)\right| \leq \eta}|f(t)|^{2} \mathrm{~d} t \leq \int_{\left|t-\mu_{p}(f)\right|>\eta}|f(t)|^{2} \mathrm{~d} t .
$$

Combining (2.7), (2.8), (2.9), (2.10) gives

$$
\frac{1}{2} \leq(1-\alpha)^{-2}\left(\frac{1}{4}+\alpha^{2}+2 \alpha\right),
$$

which yields a contradiction when $0<\alpha<\frac{\sqrt{38}}{2}-3 \approx 0.08$. Therefore, $\mid \mu_{p}\left(h_{\alpha}\right)-$ $\mu_{p}(f) \mid \leq 2 \eta$ must hold. In particular, (2.6) holds when $\alpha$ is sufficiently small. Step III. We next show that

$$
\Delta_{p}^{2}(f) \leq \liminf _{\alpha \rightarrow 0} \Delta_{p}^{2}\left(h_{\alpha}\right)
$$


Let $\alpha_{n} \rightarrow 0$ be a sequence such that $\Delta_{p}^{2}\left(h_{\alpha_{n}}\right) \rightarrow \liminf _{\alpha \rightarrow 0} \Delta_{p}^{2}\left(h_{\alpha}\right)$. As $\mu_{p}\left(h_{\alpha_{n}}\right)$ is bounded, we may further assume that $\mu_{p}\left(h_{\alpha_{n}}\right)$ converges by using a convergent subsequence. But then

$$
\begin{aligned}
\Delta_{p}^{2}(f) & \leq \int\left|t-\mu_{p}\left(h_{\alpha_{n}}\right)\right|^{2}|f(t)|^{2} \mathrm{~d} t=\int\left|t-\mu_{p}\left(h_{\alpha_{n}}\right)\right|^{p}\left|\left\|f+\alpha_{n} g\right\|_{2} h_{\alpha_{n}}(t)-\alpha_{n} g(t)\right|^{2} \mathrm{~d} t \\
& \leq\left[\left(1+\alpha_{n}\right) \Delta_{p}\left(h_{\alpha_{n}}\right)+\alpha_{n}\left(\int\left|t-\mu_{p}\left(h_{\alpha_{n}}\right)\right|^{p}|g(t)|^{2} \mathrm{~d} t\right)^{1 / 2}\right]^{2} \\
& \rightarrow \liminf _{\alpha \rightarrow 0} \Delta_{p}^{2}\left(h_{\alpha}\right),
\end{aligned}
$$

where we have used the dominated convergence theorem for the last integral. Thus, (2.11) holds, and together with (2.5) this implies the theorem's conclusion on $p$ dispersions:

$$
\lim _{\alpha \rightarrow 0} \Delta_{p}\left(h_{\alpha}\right)=\Delta_{p}(f)
$$

Step IV. Finally, we show that

$$
\lim _{\alpha \rightarrow 0} \mu_{p}\left(h_{\alpha}\right)=\mu_{p}(f) .
$$

By (2.6), $\mu_{p}\left(h_{\alpha}\right)$ is bounded for $\alpha$ small enough. It is thus enough to prove that if $\alpha_{n} \rightarrow 0$ is such that $\mu_{p}\left(h_{\alpha_{n}}\right)$ converges to some $\mu \in \mathbb{R}$, then $\mu=\mu_{p}(f)$.

Using the dominated convergence theorem, it can be shown that

$$
\Delta_{p}^{2}\left(h_{\alpha_{n}}\right)=\int\left|t-\mu_{p}\left(h_{\alpha_{n}}\right)\right|^{p}\left|h_{\alpha_{n}}(t)\right|^{2} \mathrm{~d} t \rightarrow \int|t-\mu|^{p}|f(t)|^{2} \mathrm{~d} t .
$$

On the other hand, by (2.12),$\Delta_{p}^{2}\left(h_{\alpha_{n}}\right) \rightarrow \Delta_{p}^{2}(f)$. By uniqueness of the global minimum defining $\Delta_{p}(f)$ it follows that $\mu=\mu_{p}(f)$.

\section{COMPACTNESS AND TIME-FREQUENCY CONCENTRATION}

3.1. Compactness and time-frequency concentration. We shall require the following compactness result of Kolmogorov, Riesz, and Tamarkin, [15, 20, 23]; also see [8, 26, 12, 7]. Recall that a set is relatively compact if its closure is compact.

Theorem 3.1. Let $\mathcal{K}$ be a bounded subset of $L^{2}(\mathbb{R})$. Then $\mathcal{K}$ is relatively compact if and only if the following two conditions hold:

- $\mathcal{K}$ is equicontinuous: $\int_{\mathbb{R}}|f(t+a)-f(t)|^{2} \mathrm{~d} t \rightarrow 0$ uniformly in $f \in \mathcal{K}$ as $a \rightarrow 0$,

- $\mathcal{K}$ has uniform decay: $\int_{|t| \geq R}|f(t)|^{2} \mathrm{~d} t \rightarrow 0$ uniformly in $f \in \mathcal{K}$ as $R \rightarrow+\infty$.

These properties can be reformulated in terms of the Fourier transform; see [18, 22].

Theorem 3.2. Let $\mathcal{K}$ be a bounded subset of $L^{2}(\mathbb{R})$ and let $\widehat{\mathcal{K}}=\{\widehat{f}: f \in \mathcal{K}\}$. Then $\mathcal{K}$ is equicontinuous if and only if $\widehat{\mathcal{K}}$ has uniform decay. In particular, the following conditions are equivalent:

- $\mathcal{K}$ is relatively compact,

- $\mathcal{K}$ and $\widehat{\mathcal{K}}$ are both equicontinuous,

- $\mathcal{K}$ and $\widehat{\mathcal{K}}$ both have uniform decay. 
This gives the following corollary. The result for $K_{\varphi, \psi}$ appears in [18, 22]. The result for $K_{A}^{p, q}$ appears with $p=q=2$ in [22]. Since [22] is unpublished and since we shall require the result for general values of $p, q$, we include the proof here.

Corollary 3.3. Fix $A>0, p, q>1$ and $\varphi, \psi \in L^{2}(\mathbb{R})$, and define the closed sets $\mathcal{K}_{A}^{p, q}=\left\{f \in L^{2}(\mathbb{R}):\left|\mu_{p}(f)\right| \leq A,\left|\Delta_{p}(f)\right| \leq A,\left|\mu_{q}(\widehat{f})\right| \leq A\right.$ and $\left.\left|\Delta_{q}(\widehat{f})\right| \leq A\right\}$, $\mathcal{K}_{\varphi, \psi}=\left\{f \in L^{2}(\mathbb{R}):|f(t)| \leq|\varphi(t)|\right.$ and $|\widehat{f}(t)| \leq|\psi(t)|$ for a.e. $\left.t \in \mathbb{R}\right\}$.

Then $\mathcal{K}_{A}^{p, q}$ and $\mathcal{K}_{\varphi, \psi}$ are compact subsets of $L^{2}(\mathbb{R})$.

Proof. For $\mathcal{K}_{A}^{p, q}$ note that if $|t| \geq R>2 A$ and $|a| \leq A$, then $|t-a| \geq R / 2$. It follows that if $\left|\mu_{p}(f)\right| \leq A$ and $\Delta_{p}(f) \leq A$, then as $R \rightarrow \infty$,

$$
\int_{|t| \geq R}|f(t)|^{2} \mathrm{~d} t \leq \frac{2^{p}}{R^{p}} \int_{|t| \geq R}\left|t-\mu_{p}(f)\right|^{p}|f(t)|^{2} \mathrm{~d} t \leq \frac{2^{p} A^{2}}{R^{p}} \rightarrow 0 .
$$

This, along with a similar computation for $\widehat{f}$ and Theorem $\underline{3.2}$ shows that $\mathcal{K}_{A}^{p, q}$ is compact.

For $\mathcal{K}_{\varphi, \psi}$ note that $\varphi \in L^{2}(\mathbb{R})$ implies that $\int_{|t| \geq R}|f(t)|^{2} \mathrm{~d} t \leq \int_{|t| \geq R}|\varphi(t)|^{2} \mathrm{~d} t \rightarrow$ 0 , as $R \rightarrow \infty$. This, along with a similar computation for $\widehat{f}$ and Theorem 3.2 shows that $\mathcal{K}_{\varphi, \psi}$ is compact.

Note that if $C, s>0$ and $\left\{e_{n}\right\}_{n=1}^{\infty} \subset L^{2}(\mathbb{R})$ with $\left\|e_{n}\right\|_{2}=1$ satisfies

$$
\forall f \in \overline{\operatorname{span}}\left\{e_{n}\right\}_{n=1}^{\infty}, \quad\left(\sum_{n=1}^{\infty}\left|\left\langle f, e_{n}\right\rangle\right|^{s}\right)^{1 / s} \leq C\|f\|_{2},
$$

then $\left\{e_{n}\right\}_{n=1}^{\infty}$ converges weakly to 0 . In particular, $\left\{e_{n}\right\}_{n=1}^{\infty}$ cannot be a relatively compact subset of $L^{2}(\mathbb{R})$. We thus obtain the following.

Corollary 3.4. Suppose that $\left\{e_{n}\right\}_{n=1}^{\infty} \subset L^{2}(\mathbb{R})$ with $\left\|e_{n}\right\|_{2}=1$ satisfies one of the following two conditions:

- there exists $A>0, p, q>1$ such that, for every $n \in \mathbb{N}$,

$$
\left|\mu_{p}\left(e_{n}\right)\right| \leq A, \Delta_{p}\left(e_{n}\right) \leq A,\left|\mu_{q}\left(\widehat{e_{n}}\right)\right| \leq A, \Delta_{q}\left(\widehat{e_{n}}\right) \leq A,
$$

- there exists $\varphi, \psi \in L^{2}(\mathbb{R})$ such that

$$
\left|e_{n}(t)\right| \leq|\varphi(t)| \text { and }\left|\widehat{e_{n}}(t)\right| \leq|\psi(t)| \text { a.e. } t \in \mathbb{R} .
$$

Then $\left\{e_{n}\right\}_{n=1}^{\infty}$ cannot satisfy (3.14). In particular, by (2.2) and (2.3) the system $\left\{e_{n}\right\}_{n=1}^{\infty}$ cannot be a Schauder basis or frame for its closed linear span.

3.2. Compactness and time-frequency concentration: quantitative estimates. In this section we will prove the following more quantitative version of Corollary 3.4. We use the notation $\# S$ to denote the cardinality of a set $S$.

Theorem 3.5. Fix $A, C, s>0, p, q>1$ and $\varphi, \psi \in L^{2}(\mathbb{R})$. There exist constants $N_{1}=N_{1}(A, C, s, p, q)$ and $N_{2}=N_{2}(\varphi, \psi, C, s)$ such that if $\left\{e_{n}\right\}_{n=1}^{\infty} \subset L^{2}(\mathbb{R})$ with $\left\|e_{n}\right\|_{2}=1$ satisfies (3.14), then the sets

$$
\begin{aligned}
& I=\left\{n \in \mathbb{N}:\left|\mu_{p}\left(e_{n}\right)\right| \leq A, \Delta_{p}\left(e_{n}\right) \leq A,\left|\mu_{q}\left(\widehat{e_{n}}\right)\right| \leq A, \text { and } \Delta_{q}\left(\widehat{e_{n}}\right) \leq A\right\}, \\
& J=\left\{n \in \mathbb{N}:\left|e_{n}(t)\right| \leq|\varphi(t)| \text { and }\left|\widehat{e_{n}}(t)\right| \leq|\psi(t)| \text { for a.e. } t \in \mathbb{R}\right\}
\end{aligned}
$$

satisfy $\# I \leq N_{1}$ and $\# J \leq N_{2}$. 
For example, note that Theorem 3.5 applies whenever $\left\{e_{n}\right\}_{n=1}^{\infty} \subset L^{2}(\mathbb{R})$ is a unit-norm frame or Schauder basis for $L^{2}(\mathbb{R})$. For other quantitative bounds on $N_{1}, N_{2}$ in the cases of orthonormal bases and almost orthonormal Riesz bases, see [16, 14].

Before proving Theorem 3.5 we shall need the following lemma.

Lemma 3.6. Suppose that $\left\{e_{n}\right\}_{n=1}^{k} \subset L^{2}(\mathbb{R})$ with $\left\|e_{n}\right\|_{2}=1$ is such that for every $m \in\{1,2, \ldots, k\}$,

$$
\#\left\{n \in\{1,2, \ldots, k\}:\left|\left\langle e_{n}, e_{m}\right\rangle\right| \geq 1 / 2\right\} \leq D .
$$

There exists $T \subset\{1,2, \ldots, k\}$ satisfying:

- $\# T \geq\left\lfloor\frac{k+\lceil D\rceil}{\lceil D\rceil+1}\right\rfloor$.

- If $m, n \in T$ and $m \neq n$, then $\left|\left\langle e_{m}, e_{n}\right\rangle\right|<1 / 2$.

Proof. We may without loss of generality assume that $D$ is an integer by replacing $D$ with $\lceil D\rceil$. Reordering $\left\{e_{n}\right\}_{n=1}^{k}$ if necessary, we may assume that $\left|\left\langle e_{n}, e_{1}\right\rangle\right|<$ $1 / 2$ for $n=2, \ldots, k-D$. Reordering $\left\{e_{n}\right\}_{n=2}^{k-D}$ if necessary, we may assume that $\left|\left\langle e_{n}, e_{2}\right\rangle\right|<1 / 2$ for $n=3, \ldots, k-2 D$. Continuing this process, we may assume that $\left|\left\langle e_{n}, e_{j}\right\rangle\right|<1 / 2$ for $n=(j+1), \ldots,(k-j D)$ as long as $j+1 \leq k-j D$, i.e., $j+1 \leq$ $(k+D) /(D+1)$. This process thus stops when $M:=j+1=\lfloor(k+D) /(D+1)\rfloor$ and provides a set $\left\{e_{m}\right\}_{m=1}^{M}$ that satisfies $\left|\left\langle e_{m}, e_{n}\right\rangle\right|<1 / 2$ whenever $m \neq n$ and $1 \leq m, n \leq M$.

We are now ready to prove Theorem 3.5 .

Proof of Theorem 3.5. We shall only prove the bound for \#I since the argument for \# $J$ is similar. It follows from Corollary 3.4 that the set $I$ is finite. Thus, by reordering the sequence $\left\{e_{n}\right\}_{n=1}^{\infty}$, we may assume that $I=\{1, \ldots, k\}$. We want to prove that there is a constant $N=N(A, C, s, p, q)$ such that $k \leq N$.

By (3.14), for all $m \in \mathbb{N}$ we have $\sum_{n=1}^{\infty}\left|\left\langle e_{m}, e_{n}\right\rangle\right|^{s} \leq C^{s}$. Tchebyshev's inequality gives

$$
\forall m \in \mathbb{N}, \quad \#\left\{n \in \mathbb{N}:\left|\left\langle e_{m}, e_{n}\right\rangle\right| \geq 1 / 2\right\} \leq 2^{s} C^{s} .
$$

Let $T \subset\{1,2, \ldots, k\}$ be the set obtained when Lemma 3.6 is applied to $\left\{e_{n}\right\}_{n=1}^{k}$ with $D=2^{s} C^{s}$. Since $\# T \geq\lfloor(k+\lceil D\rceil) /(\lceil D\rceil+1)\rfloor$, it will suffice to prove that there is a constant $L=L(A, C, s, p, q)$ such that $\# T \leq L$.

Since $\mathcal{K}=\mathcal{K}_{A}^{p, q}$ is relatively compact (by Corollary $\left[3.3\right.$ ), the $L^{2}(\mathbb{R})$ closure of $\mathcal{K}$, denoted $\overline{\mathcal{K}}$, is compact. The open balls $B_{1 / 2}(x)=\left\{y \in L^{2}(\mathbb{R}):\|y-x\|_{2}<1 / 2\right\}$ with $x \in \mathcal{K}$ provide an open cover of $\overline{\mathcal{K}}$. So there exist $\left\{x_{n}\right\}_{n=1}^{M} \subset \mathcal{K}$ such that $\overline{\mathcal{K}} \subset \bigcup_{n=1}^{M} B_{1 / 2}\left(x_{n}\right)$. By properties of the set $T$, if $m \neq n$ and $m, n \in T$, then

$$
\left\|e_{n}-e_{m}\right\|_{2}^{2}=2\left[1-\Re\left(\left\langle e_{n}, e_{m}\right\rangle\right)\right] \geq 2\left(1-\left|\left\langle e_{n}, e_{m}\right\rangle\right|\right)>1 .
$$

So each $B_{1 / 2}\left(x_{n}\right)$ contains at most one of the $\left\{e_{n}\right\}_{n \in T}$. However, since

$$
\left\{e_{n}\right\}_{n \in T} \subset \overline{\mathcal{K}} \subset \bigcup_{n=1}^{M} B_{1 / 2}\left(x_{n}\right),
$$

we obtain $\# T \leq M$. Thus, $k \leq M(\lceil D\rceil+1)+1$ and we may take $L=M(\lceil D\rceil+$ 1) +1 . 
Note that if $\left\{e_{n}\right\}_{n=1}^{\infty} \subset L^{2}(\mathbb{R})$ satisfies (3.14), then $\left\{f_{n}\right\}_{n=1}^{\infty}$ defined by $f_{n}(t)=$ $e^{-2 \pi i \mu t} e_{n}(t)$ with fixed $\mu \in \mathbb{R}$ also satisfies (3.14). Also, $\mu_{p}\left(f_{n}\right)=\mu_{p}\left(e_{n}\right), \mu_{q}\left(\widehat{f_{n}}\right)=$ $\mu_{q}\left(\widehat{e_{n}}\right)-\mu, \Delta\left(f_{n}\right)=\Delta\left(e_{n}\right), \Delta\left(\widehat{f_{n}}\right)=\Delta\left(\widehat{e_{n}}\right)$. Consequently, for every $\mu \in \mathbb{R}$,

$\#\left\{n \in \mathbb{N}:\left|\mu_{p}\left(e_{n}\right)\right| \leq A, \Delta_{p}\left(e_{n}\right) \leq A,\left|\mu_{q}\left(\widehat{e_{n}}\right)-\mu\right| \leq A, \Delta_{q}\left(\widehat{e_{n}}\right) \leq A\right\} \leq N_{1}$,

where the constant $N_{1}$ is from Theorem 3.5 and does not depend on $\mu$. This implies the following corollary.

Corollary 3.7. Fix $A>0$ and $p, q>1$. Suppose that $\left\{e_{n}\right\}_{n=1}^{\infty} \subset L^{2}(\mathbb{R})$ with $\left\|e_{n}\right\|_{2}=1$ satisfies (3.14) and that, for every $n \in \mathbb{N}$,

$$
\left|\mu_{p}\left(e_{n}\right)\right| \leq A, \quad \Delta_{p}\left(e_{n}\right) \leq A, \quad \Delta_{q}\left(\widehat{e_{n}}\right) \leq A .
$$

Then one may reorder the sequence $\left\{e_{n}\right\}_{n=1}^{\infty}$ so that there exist constants $b, c>0$ for which $\left|\mu_{q}\left(\widehat{e_{n}}\right)\right| \geq($ cn $-b)$ holds for all $n \in \mathbb{N}$.

\section{ONE BOUNDED MEAN AND TWO BOUNDED VARIANCES}

The following lemma is a straightforward extension of Lemma 2 in [19].

Lemma 4.1. Fix $p, q>1$. Suppose $f, g \in L^{2}(\mathbb{R})$ and $\|f\|_{2}=\|g\|_{2}=1$ and that the dispersions $\Delta_{p}(f), \Delta_{q}(\widehat{f}), \Delta_{p}(g), \Delta_{q}(\widehat{g})$ are all finite. Then

$$
|\langle f, g\rangle| \leq \frac{2^{p / 2}\left(\Delta_{p}(f)+\Delta_{p}(g)\right)+2^{q / 2}\left(\Delta_{q}(\widehat{f})+\Delta_{q}(\widehat{g})\right)}{\left|\mu_{p}(f)-\mu_{p}(g)\right|^{p / 2}+\left|\mu_{q}(\widehat{f})-\mu_{q}(\widehat{g})\right|^{q / 2}} .
$$

We are now in a position to prove the following theorem.

Theorem 4.2. Fix $A, B, C>0, p, q>1$ and $1<r \leq 2 \leq s<\infty$. Let $\left\{e_{n}\right\}_{n=1}^{\infty} \subset$ $L^{2}(\mathbb{R})$ with $\left\|e_{n}\right\|_{2}=1$ satisfy

$$
\forall f \in L^{2}(\mathbb{R}), \quad B\left(\sum_{n=1}^{\infty}\left|\left\langle f, e_{n}\right\rangle\right|^{s}\right)^{1 / s} \leq\|f\|_{2} \leq C\left(\sum_{n=1}^{\infty}\left|\left\langle f, e_{n}\right\rangle\right|^{r}\right)^{1 / r} .
$$

If $q r>2$, then it is not possible for $\left\{e_{n}\right\}_{n=1}^{\infty}$ to satisfy

$$
\forall n \in \mathbb{N}, \quad\left|\mu_{p}\left(e_{n}\right)\right| \leq A,\left|\Delta_{p}\left(e_{n}\right)\right| \leq A, \text { and }\left|\Delta_{q}\left(\widehat{e_{n}}\right)\right| \leq A .
$$

Proof. To get a contradiction suppose that (4.16) holds. Note that (4.15) is still valid after reordering the sequence $\left\{e_{n}\right\}_{n=1}^{\infty}$. By Corollary 3.7 we may thus assume that there exist constants $b, c>0$ such that $\left|\mu\left(\widehat{e}_{n}\right)\right| \geq(c n-b)$ holds for all $n \in \mathbb{N}$.

Let $g(x)=2^{1 / 4} e^{-\pi x^{2}}$ and $g_{N}(x)=g(x-N)$. Note that $\|g\|_{2}=1$. By Lemma2.1 we have $\mu_{p}\left(g_{N}\right)=\mu_{p}(g)+N=N, \mu_{q}\left(\widehat{g_{N}}\right)=\mu_{q}(\widehat{g})=0, \Delta_{p}\left(g_{N}\right)=\Delta_{p}(g), \Delta_{q}\left(\widehat{g_{N}}\right)=$ $\Delta_{q}(\widehat{g})$. By Lemma 4.1, for $N>A$,

$$
\left|\left\langle g_{N}, e_{n}\right\rangle\right| \leq \frac{2^{p / 2}\left(\Delta_{p}(g)+A\right)+2^{q / 2}\left(\Delta_{q}(\widehat{g})+A\right)}{|N-A|^{p / 2}+|c n-b|^{q / 2}} .
$$

Hence, for an appropriate constant $c_{1}>0$,

$$
1=\left\|g_{N}\right\|_{2}^{r} \leq C^{r} \sum_{n=1}^{\infty}\left|\left\langle g_{N}, e_{n}\right\rangle\right|^{r} \leq c_{1} \sum_{n=1}^{\infty} \frac{1}{\left(|N-A|^{p / 2}+|c n-b|^{q / 2}\right)^{r}} .
$$

This gives a contradiction since the assumptions $q r>2$ and $p>0$ imply that

$$
\lim _{N \rightarrow \infty} \sum_{n=1}^{\infty} \frac{1}{\left(|N-A|^{p / 2}+|c n-b|^{q / 2}\right)^{r}}=0
$$


It would be interesting to know whether the condition $q r>2$ is necessary.

We obtain the following corollaries as direct consequences of (2.2) and (2.3).

Corollary 4.3. Fix $A>0, p>1$. If $\left\{e_{n}\right\}_{n=1}^{\infty} \subset L^{2}(\mathbb{R})$ satisfies $\left\|e_{n}\right\|_{2}=1$ and

$$
\forall n \in \mathbb{N}, \quad\left|\mu_{p}\left(e_{n}\right)\right| \leq A,\left|\Delta_{p}\left(e_{n}\right)\right| \leq A, \text { and }\left|\Delta_{2}\left(\widehat{e_{n}}\right)\right| \leq A,
$$

then $\left\{e_{n}\right\}_{n=1}^{\infty}$ cannot be a Schauder basis for $L^{2}(\mathbb{R})$.

Corollary 4.4. Fix $A>0, p, q>1$. If $\left\{e_{n}\right\}_{n=1}^{\infty} \subset L^{2}(\mathbb{R})$ satisfies $\left\|e_{n}\right\|_{2}=1$ and $\forall n \in \mathbb{N}, \quad\left|\mu_{p}\left(e_{n}\right)\right| \leq A,\left|\Delta_{p}\left(e_{n}\right)\right| \leq A, \quad$ and $\quad\left|\Delta_{q}\left(\widehat{e_{n}}\right)\right| \leq A$, then $\left\{e_{n}\right\}_{n=1}^{\infty}$ cannot be a frame for $L^{2}(\mathbb{R})$.

\section{EXACT SYSTEMS AND TIME-FREQUENCY LOCALIZATION}

In this section we shall show that the phenomenon described in Theorem 4.2 does not hold for exact systems in $L^{2}(\mathbb{R})$. We shall make use of the following example.

Example 5.1. Let $g(t)=2^{1 / 4} e^{-\pi t^{2}}$ and consider the Gabor system $\mathcal{G}(g, 1,1)=$ $\left\{g_{m, n}\right\}_{m, n \in \mathbb{Z}}$ defined by

$$
\forall m, n, \in \mathbb{Z}, \quad g_{m, n}(t)=e^{2 \pi i m t} g(t-n) .
$$

The unit-norm system $\mathcal{G}_{0}=\mathcal{G}(g, 1,1) \backslash\left\{g_{1,1}\right\}$ is an exact system in $L^{2}(\mathbb{R})$; see 9 . Moreover, $\mathcal{G}_{0}$ is neither a frame nor a Schauder basis for $L^{2}(\mathbb{R})$.

Further related examples and uncertainty principles involving exact Gabor systems can be found in [1] and [11, 17] respectively.

The construction in the following theorem is inspired by [3, 25].

Theorem 5.2. Fix $p, q>1$ and let $g(t)=2^{1 / 4} e^{-\pi t^{2}}$. Given $\epsilon>0$, there exists a unit-norm exact system $\left\{f_{n}\right\}_{n=1}^{\infty}$ in $L^{2}(\mathbb{R})$ such that

$\forall n \in \mathbb{N}, \quad\left|\mu_{p}\left(f_{n}\right)\right|<\epsilon, \quad\left|\mu_{q}\left(\widehat{f_{n}}\right)\right|<\epsilon, \quad \Delta_{p}\left(f_{n}\right)<\Delta_{p}(g)+\epsilon, \quad \Delta_{q}\left(\widehat{f_{n}}\right)<\Delta_{q}(\widehat{g})+\epsilon$, and such that $\varphi(t)=\sup _{n \in \mathbb{N}}\left|f_{n}(t)\right|$ and $\psi(\xi)=\sup _{n \in \mathbb{N}}\left|\widehat{f_{n}}(\xi)\right|$ satisfy $\varphi, \psi \in L^{2}(\mathbb{R})$.

Proof. Note that the system $\mathcal{G}(g, 1,1)$ from Example [5.1 satisfies $\|g\|_{2}=1$ and that $\mu_{p}\left(g_{m, n}\right)=\mu_{p}(g)+n=n, \mu_{q}\left(\widehat{g_{m, n}}\right)=m, \Delta_{p}\left(g_{m, n}\right)=\Delta_{p}(g)$, and $\Delta_{q}\left(\widehat{g_{m, n}}\right)=$ $\Delta_{q}(\widehat{g})$. We may enumerate the system $\mathcal{G}_{0}$ from Example 5.1 as $\mathcal{G}_{0}=\left\{e_{n}\right\}_{n=1}^{\infty}$ so that $e_{1}=g_{0,0}=g$.

Let $\left\{\alpha_{n}\right\}_{n=1}^{\infty} \subset \mathbb{R}$ satisfying $0<\alpha_{n}<1$ be a sequence to be specified below and define

Note that

$$
\forall n \in \mathbb{N}, \quad f_{n}=\frac{e_{1}+\alpha_{n} e_{n+1}}{\left\|e_{1}+\alpha_{n} e_{n+1}\right\|_{2}} .
$$

$$
\widehat{f_{n}}=\frac{\widehat{e_{1}}+\alpha_{n} \widehat{e_{n+1}}}{\left\|\widehat{e_{1}}+\alpha_{n} \widehat{e_{n+1}}\right\|_{2}} \text {. }
$$

Using Proposition 2.2. for each $n \in \mathbb{N}$ we select $\alpha_{n}$ sufficiently small so that $0<$ $\alpha_{n}<2^{-n}$ and such that

$$
\left|\mu_{p}\left(f_{n}\right)\right|=\left|\mu_{p}\left(f_{n}\right)-\mu_{p}\left(e_{1}\right)\right|<\epsilon \quad \text { and } \quad\left|\mu_{q}\left(\widehat{f_{n}}\right)\right|=\left|\mu_{q}\left(\widehat{f_{n}}\right)-\mu_{q}\left(\widehat{e_{1}}\right)\right|<\epsilon,
$$

and

$$
\Delta_{p}\left(f_{n}\right)<\Delta_{p}\left(e_{1}\right)+\epsilon \quad \text { and } \quad \Delta_{q}\left(\widehat{f_{n}}\right)<\Delta_{q}\left(\widehat{e_{1}}\right)+\epsilon .
$$


Further, since $1 / 2 \leq 1-2^{-n}<1-\alpha_{n} \leq\left\|e_{1}+\alpha_{n} e_{n+1}\right\|_{2}$ we have $\left|f_{n}\right| \leq 2\left(\left|e_{1}\right|+\right.$ $\left.2^{-n}\left|e_{n+1}\right|\right)$ and hence $|\varphi| \leq 2\left|e_{1}\right|+2 \sum_{n=1}^{\infty} 2^{-n}\left|e_{n+1}\right|$. Thus $\left\|e_{n}\right\|_{2}=1$ gives

$$
\|\varphi\|_{2} \leq 2\left\|e_{1}\right\|_{2}+2 \sum_{n=1}^{\infty} 2^{-n}\left\|e_{n+1}\right\|_{2} \leq 4
$$

Hence $\varphi \in L^{2}(\mathbb{R})$. A similar computation shows that $\psi \in L^{2}(\mathbb{R})$.

Finally, to show that $\left\{f_{n}\right\}_{n=1}^{\infty}$ is exact we must show that it is complete and minimal. First, note that for each $n \in \mathbb{N}, e_{n} \in \overline{\operatorname{span}}\left\{f_{n}\right\}_{n=1}^{\infty}$. Indeed, $\| e_{1}+$ $\alpha_{n} e_{n+1} \|_{2} f_{n}=e_{1}+\alpha_{n} e_{n+1} \rightarrow e_{1}$ as $n \rightarrow \infty$, and also for each $n \in \mathbb{N}, e_{n+1}=$ $\frac{1}{\alpha_{n}}\left(\left\|e_{1}+\alpha_{n} e_{n+1}\right\|_{2} f_{n}-e_{1}\right)$. Thus, $L^{2}(\mathbb{R})=\overline{\operatorname{span}}\left\{e_{n}\right\}_{n=1}^{\infty} \subset \overline{\operatorname{span}}\left\{f_{n}\right\}_{n=1}^{\infty}$, and $\left\{f_{n}\right\}_{n=1}^{\infty}$ is complete in $L^{2}(\mathbb{R})$.

To see that $\left\{f_{n}\right\}_{n=1}^{\infty}$ is minimal suppose that $f_{N} \in \overline{\operatorname{span}}\left\{f_{n}: n \neq N\right\}$. Since, as above, $e_{1} \in \operatorname{span}\left\{f_{n}: n>N\right\}$, it would follow that $e_{N+1} \in \overline{\operatorname{span}}\left\{f_{n}: n \neq N\right\}$. However this contradicts the minimality of $\left\{e_{n}\right\}_{n=1}^{\infty}$ since $\operatorname{span}\left\{f_{n}: n \neq N\right\} \subset$ $\overline{\operatorname{span}}\left\{e_{n}: n \neq(N+1)\right\}$ and $e_{N+1} \notin \overline{\operatorname{span}}\left\{e_{n}: n \neq(N+1)\right\}$.

By Corollary 4.3 the exact system $\left\{f_{n}\right\}_{n=1}^{\infty}$ in Theorem $[5.2$ is not a Schauder basis.

\section{ACKNOWLEDGMENTS}

The authors thank S. Nitzan and H. Shapiro for helpful discussions related to the material and are thankful for the anonymous referee's careful remarks which improved the paper.

\section{REFERENCES}

1. G. Ascensi, Y. Lyubarskii and K. Seip, Phase space distribution of Gabor expansions, Appl. Comput. Harmon. Anal. 26 (2009), 277-282. MR2490219 (2010b:42039)

2. J. J. Benedetto, C. Heil and D. F. Walnut, Differentiation and the Balian-Low theorem. J. Fourier Anal. Appl. 1 (1995), 355-402. MR1350699 (96f:42002)

3. J. Bourgain, A remark on the uncertainty principle for Hilbertian basis, J. Funct. Anal., 79 (1988), 136-143. MR950087 (89f:81025)

4. P. Casazza, The art of frame theory, Taiwanese J. Math. 4 (2000), 129-201. MR1757401 (2001f:42046)

5. P. Casazza, O. Christensen and D. Stoeva, Frame expansions in separable Banach spaces, J. Math. Anal. Appl., 307 (2005), 710-723. MR2142455 (2006e:42044)

6. O. Christensen, An Introduction to Frames and Riesz Bases, Birkhäuser, Boston, 2003. MR 1946982 (2003k:42001)

7. M. Dörfler, K. Gröchenig and H. G. Feichtinger, Compactness criteria in function spaces, Coll. Math. 94 (2002), 37-50. MR1930200 (2003j:46041)

8. N. Dunford and J. T. Schwartz, Linear Operators, Part 1, Wiley-Interscience, New York, 1966. MR0117523 (22:8302)

9. G. B. Folland, Harmonic Analysis on Phase Space, Annals of Mathematics Studies, Princeton Univ. Press, Princeton, NJ, 1989. MR983366 (92k:22017)

10. G. B. Folland and A. Sitaram, The uncertainty principle: A mathematical survey, J. Fourier Anal. Appl. 3 (1997), 207-238. MR.1448337 (98f:42006)

11. C. Heil and A. M. Powell, Regularity for complete and minimal Gabor systems on a lattice, Illinois J. Math., 53 (2009), 1077-1094.

12. H. Hanche-Olsen and H. Holden, The Kolmogorov-Riesz compactness theorem, Expo. Math. 28 (2010), 385-394.

13. V. I. Gurariǔ and N. I. Gurariŭ, Bases in uniformly convex and uniformly smooth Banach spaces (Russian), Izv. Akad. Nauk SSSR Ser. Mat. 35 (1971), 210-215. MR0283549 (44:780)

14. P. Jaming and A. M. Powell, Uncertainty principles for orthonormal sequences, J. Funct. Anal. 243 (2007), 611-630. MR.2289698(2008b:42011) 
15. A. N. Kolmogorov, Über Kompaktheit der Funktionenmengen bei der Konvergenz im Mittel, Nachr. Ges. Wiss. Göttingen 9 (1931), 60-63.

16. E. Malinnikova, Orthonormal sequences in $L^{2}\left(R^{d}\right)$ and time frequency localization, Jour. Fourier. Anal. Appl. 16 (2010), 983-1006.

17. S. Nitzan and J.-F. Olsen, From exact sequences to Riesz bases in the Balian-Low theorem, accepted to appear in Jour. Fourier Anal. Appl., DOI 10.100/s00041-010-9150-5.

18. R. L. Pego, Compactness in $L^{2}$ and the Fourier transform. Proc. Amer. Math. Soc. 95 (1985), 252-254. MR801333 (87f:42025)

19. A. M. Powell, Time-frequency mean and variance sequences of orthonormal bases, Jour. Fourier. Anal. Appl. 11 (2005), 375-387. MR2169472 (2006g:42009)

20. M. Riesz, Sur les ensembles compacts de fonctions sommables, Acta Szeged Sect. Math. 6 (1933), 136-142.

21. W. H. Ruckle, The extent of the sequence space associated with a basis, Canad. J. Math. 24 (1972), 636-641. MR0300057 (45:9105)

22. H. S. Shapiro, Uncertainty principles for bases in $L^{2}(\mathbb{R})$, unpublished manuscript (1991).

23. J. D. Tamarkin, On the compactness of the space $L^{p}$, Bull. Amer. Math. Soc. 32 (1932), 79-84. MR 1562331

24. R. Young, An Introduction to Nonharmonic Fourier Series, revised first ed., Academic Press, San Diego, 2001. MR.1836633 (2002b:42001)

25. R. Young, On complete biorthogonal systems, Proc. Amer. Math. Soc. 83 (1981), 537-540. MR 627686 (84c:42048)

26. K. Yoshida, Functional Analysis, second ed., Springer Verlag, New York, 1968. MR0239384 $(39: 741)$

Faculté des Sciences, MAPMO, Université d'Orléans, BP 6759, F 45067 Orléans Cedex 2, France

Current address: Institut de Mathématiques de Bordeaux UMR 5251, Université Bordeaux 1, cours de la Libération, F 33405 Talence cedex, France

E-mail address: Philippe.Jaming@gmail.com

Department of Mathematics, Vanderbilt University, Nashville, Tennessee 37240

E-mail address: alexander.m.powell@vanderbilt.edu 